Article

\title{
Condition Assessment of Paper Insulation in Oil-Immersed Power Transformers Based on the Iterative Inversion of Resistivity
}

\author{
Jiangjun Ruan ${ }^{1}$, Shuo Jin ${ }^{1, *}$, Zhiye Du ${ }^{1}$, Yiming Xie ${ }^{1}$, Lin Zhu ${ }^{2}$, Yu Tian ${ }^{1}$, Ruohan Gong ${ }^{1}$, \\ Guannan $\mathrm{Li}^{1}$ and Min Xiong ${ }^{1}$ \\ 1 School of Electrical Engineering, Wuhan University, Wuhan 430072, China; ruan308@126.com (J.R.); \\ duzhiye@126.com (Z.D.); xieyiming@whu.edu.cn (Y.X.); yu_tian@whu.edu.cn (Y.T.); \\ grhdqt@foxmail.com (R.G.); mikelifly@gmail.com (G.L.); 13419538038@163.com (M.X.) \\ 2 State Grid Hubei Electric Power Company Technology Training Center, Wuhan 430079, China; \\ zhulinwhu@163.com \\ * Correspondence: jinshuo_whu@hotmail.com
}

Academic Editor: Dongsheng Wen

Received: 14 November 2016; Accepted: 6 April 2017; Published: 10 April 2017

\begin{abstract}
The resistivity of oil impregnated paper will decrease during its aging process. This paper takes paper resistivity as an assessment index to evaluate the insulation condition of oil impregnated paper in power transformer. The feasibility of this method are discussed in two aspects: reliability and sensitivity. Iterative inversion of paper resistivity was combined with finite element simulation. Both the bisection method and Newton's method were used as iterative methods. After the analysis and comparison, Newton's method was selected as the first option of paper resistivity iteration for its faster convergence. In order to consider the spatial distribution characteristic of paper aging and enhance the calculation accuracy, the resistivity calculation is expanded to a multivariate iteration based on Newton's method, in order to consider the spatial distribution characteristic of paper aging and improve the calculation accuracy. This paper presents an exploratory research on condition assessment of oil impregnated paper insulation, and provides some reference to the security and economy operation of power transformers.
\end{abstract}

Keywords: condition assessment; oil impregnated paper; resistivity; iteration

\section{Introduction}

As the main dielectric media in oil-immersed transformers, the oil and oil impregnated paper will degrade with time, under thermal, electrical, chemical stresses and other factors [1-4]. Oil in transformers can be replaced after serious deterioration, because of its mobility. On the contrary, the replacement of oil impregnated paper is rather difficult when it degrades seriously, especially the paper covering windings. Hence, the operation of a transformer is dependent on the condition of the paper insulation [5-10] and assessing the condition of paper insulation is vital to the security and economy of the transformer's operation and even the entire power system.

Cellulose is the main component of paper. The mechanical properties of cellulose will deteriorate during its aging process. Determining the degree of polymerization (DP) value of cellulose is a standard method of quantifying cellulose degradation [4,11]. Many researches on the elapsed life estimation of power transformers were based on the regularity of DP change [12-14]. However, DP of paper inside a transformer is not easily assessable, and even destructive.

The degradation by-products of cellulose, such as hydrocarbons, acids, carbon monoxide, carbon dioxide and furfurals remain dissolved in the oil [15-18]. The contents of these by-products 
in oil can provide some information on cellulose ageing. Accordingly, dissolved gas analysis (DGA), furanic compound analysis and other by-products analysis methods have been developed and widely accepted $[11,17,19,20]$. To increase the efficiency and accuracy of diagnosis, modern technologies such as Fourier transform infrared (FTIR) spectroscopy, UV-visible spectroscopy, artificial intelligence techniques, etc. have been applied in recent years [21-25]. The major limitation of the by-product analysis methods is that they are affected by any oil replacement or oil reconditioning progress $[25,26]$.

In recent years, electrical diagnostic methods also drew the attention of many researchers. Dielectric response methods were developed as complements to the classical insulation resistance, dielectric loss factor and polarization index measurements. There are three notable methods to measure the transformer dielectric response, namely frequency domain spectroscopy (FDS), polarization and depolarization current (PDC) and return voltage measurement (RVM) [2,27-30]. The first method is based on frequency domain analysis and the other two methods are based on time domain analysis. Expert systems, grey correlation analysis, and other data analysis techniques were also applied to improve the diagnostic accuracy [31]. At the present stage, the electrical diagnostic methods mainly rely on the comparison between measured data and historical data, and may be influenced by the geometry [32]. Among these methods, there is no unified assessment index currently available for different types of transformers.

Actually, the ageing of cellulose paper is not uniform due to the existence of temperature gradients and electric gradients. Generally speaking, the above non-destructive chemical-based diagnostic techniques and electrical-based diagnostic techniques regard the solid insulation in transformers as a whole. The returned information of these methods reflects the "mean" condition of paper. From this point of view, if the spatial distribution characteristics of paper ageing were considered, the sensitivity and accuracy of paper condition assessment would be improved.

During aging processes, the reduction in the polymer chain length of cellulose contributes to the changes in the dielectric properties of cellulose paper [33,34]. Changes in the moisture and oil contents during the aging process also lead to changes in the dielectric properties of paper. These changes are the fundamental basis of all electrical diagnostic methods $[28,32,35,36]$. The basic dielectric properties for insulation are permittivity and resistivity. The resistivity of paper will decrease over time as the moisture content and aging increase [28,34], hence the resistivity of paper can be used to assess the insulation condition of oil impregnated paper.

In this paper, the resistivity of paper is used as the assessment index for the condition assessment of paper insulation in a power transformer. As resistivity is the intrinsic property of material, it is independent from the geometry or the structure of insulation. Hence a new quantitative condition assessment method based on paper resistivity can be realized, to diagnose the paper insulation condition in different transformers. It deserves to be mentioned that, considering the similar influence of ageing and moisture on paper resistivity, the presented work in this paper will not distinguish between these two factors.

According to the idea of taking paper resistivity as an assessment index, the feasibility is discussed firstly in this paper. Thereafter, calculation methods to obtain the resistivity value of paper are proposed, in order to avoid destructive sampling. The basic idea of the calculation method is as following: firstly we build the relationship between insulation resistance and paper resistivity through finite element simulation. Secondly, we iteratively calculate the paper resistivity using the measured insulation resistance. Non-uniform ageing was also considered in the iterative method to improve the accuracy of the resistivity calculations.

\section{Feasibility Analysis}

\subsection{The Ageing Condition and Resistivity of Paper}

During the aging process, the physical and chemical properties of paper will change. As a result, the resistivity of oil impregnated paper will decrease. In [37], the author provided measured resistivities 
and DP values of paper samples impregnated with different kinds of oil under different ageing temperatures, as shown in Figure 1. Oil No. 25 and oil No. 45 are two kinds of commonly used transformer oils with different melting points. The two kinds of oil have different electric characteristics. Generally speaking, oil No. 25 has lower resistivity than oil No. 45, for its higher content of aromatic hydrocarbon.

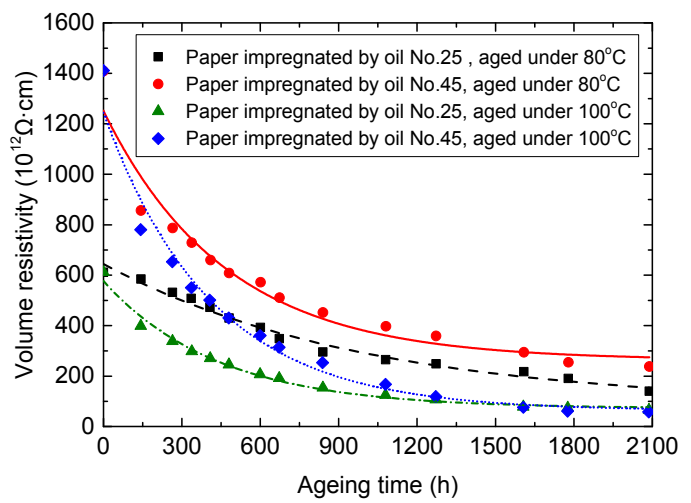

(a)

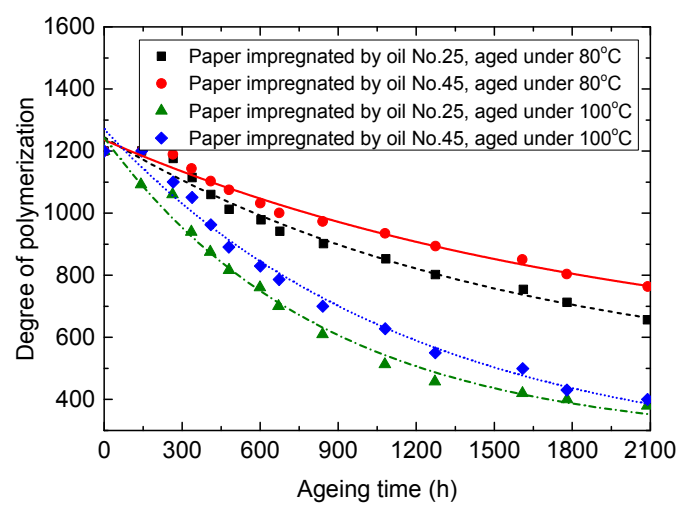

(b)

Figure 1. Measured resistivities and DP values at different ageing stages in [37]. (a) Measured resistivities under different ageing stage; (b) Measured DP values under different ageing stage.

With the measured data in Figure 1, relationships between degree of polymerization and volume resistivity of oil impregnated paper were built, as shown in Figure 2. There are good power function relationships between the degree of polymerization and volume resistivity for different kinds of paper. Table 1 provided the parameters of the power fitting curves.

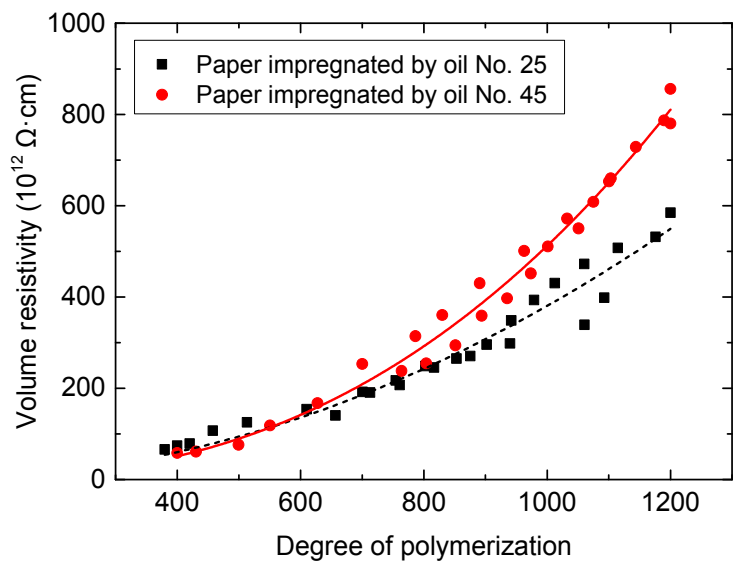

Figure 2. Relationship between DP and volume resistivity.

Table 1. Fitting information (Fitting equation: $\rho=A \times D P^{k}$ ).

\begin{tabular}{cccc}
\hline Type of Paper & $\boldsymbol{A}$ & $\boldsymbol{k}$ & $\boldsymbol{R}^{\mathbf{2}}$ (Goodness of Fit) \\
\hline Paper impregnated by oil No. 25 & $3.434 \times 10^{-4}$ & 2.015 & 0.957 \\
Paper impregnated by oil No. 45 & $1.434 \times 10^{-5}$ & 2.518 & 0.986 \\
\hline
\end{tabular}

Currently, DP is the most reliable parameter for the ageing condition assessment of oil impregnated paper. In Table 1, it is shown that volume resistivity has a good power function relationship with DP, hence volume resistivity could also efficiently reflect the ageing condition of paper. 


\subsection{The Sensitivity of Resistivity as an Assessment Index}

Under normal conditions, the permittivity of paper is one or two times larger than the permittivity of oil. Under a relatively high frequency AC voltage, the electric field in oil is stronger [38]. On the contrary, the resistivity of paper is larger than the resistivity of oil by almost two orders of magnitude. The electric field in oil impregnated paper is much stronger than that in oil under DC voltage conditions, hence, the change of paper resistivity value will induce obvious changes in measured voltages or currents under a DC electric field.

In a transformer, the main electric field component applied on the oil impregnated paper is perpendicular to its surface. Besides, in most areas of each piece of paper, the electric field distribution is relatively uniform. Therefore, a certain area of each paper can be regarded as some smaller areas of paper connected in parallel with each other. The resistivity change of a small local area will lead to an obvious change in the average resistivity of a relatively larger area.

Figure $3 \mathrm{a}$ is an idealized model of this condition. In this model, a piece of paper with an area of $S$ is under a uniform electric field. The resistivity of this paper under normal conditions is $\rho_{n}$. In this piece of paper, there is a partial ageing region with an area of $S_{a}$. The resistivity of the ageing area is $\rho_{\mathrm{a}}$. Under an electric field perpendicular to the surface, the normal area and ageing area are arranged in parallel, as shown in Figure $3 b$. The resistance expressions of each area are shown by Equations (1) and (2).

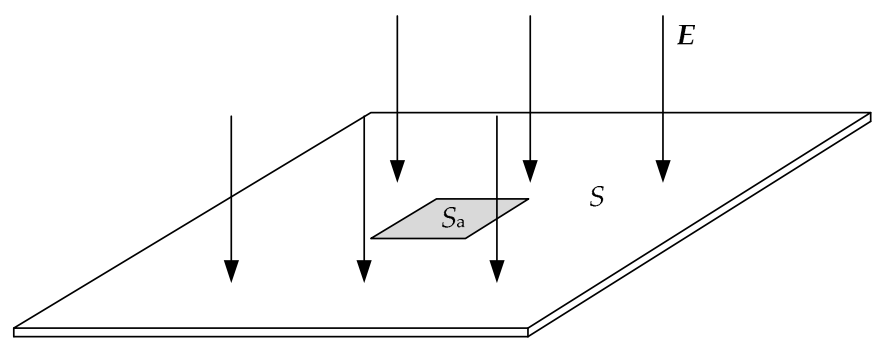

(a)

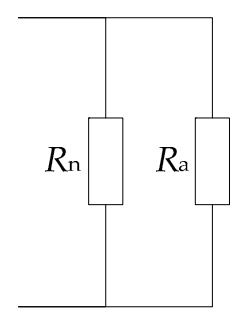

(b)

Figure 3. Idealized model of partial paper ageing. (a) Idealized model; (b) Equivalent circuit diagram.

$$
\begin{gathered}
R_{\mathrm{n}}=\frac{\rho_{\mathrm{n}} d}{\left(S-S_{\mathrm{a}}\right)} \\
R_{\mathrm{a}}=\frac{\rho_{\mathrm{a}} d}{S_{\mathrm{a}}}
\end{gathered}
$$

where $d$ is the thickness of this piece of paper.

Hence, the total resistance $R_{\text {total }}$ can be expressed as:

$$
R_{\text {total }}=\frac{R_{\mathrm{n}} R_{\mathrm{a}}}{\left(R_{\mathrm{n}}+R_{\mathrm{a}}\right)}=\frac{\rho_{\mathrm{n}} \rho_{\mathrm{a}} d}{\rho_{\mathrm{n}} S_{\mathrm{a}}+\rho_{\mathrm{a}}\left(S-S_{\mathrm{a}}\right)}
$$

Therefore, the average resistivity of the whole piece of paper can be expressed as:

$$
\rho_{\mathrm{av}}=\frac{R_{\mathrm{total}} S}{d}=\frac{\rho_{\mathrm{n}} \rho_{\mathrm{a}} S}{\rho_{\mathrm{n}} S_{\mathrm{a}}+\rho_{\mathrm{a}}\left(S-S_{\mathrm{a}}\right)}
$$

Figure 4 shows the decrease of the average resistivity with different percentages of the partial ageing region according to (4). It is obvious in Figure 4 that when $\rho_{\mathrm{a}}$ dropped to $1 \%$ of $\rho_{\mathrm{n}}$, ageing region with only $10 \%$ of the area of the whole piece of paper can reduce the average resistivity by more than $90 \%$. Therefore, even the average resistivity can sensitively reflect partial ageing in a relative larger piece of paper. 


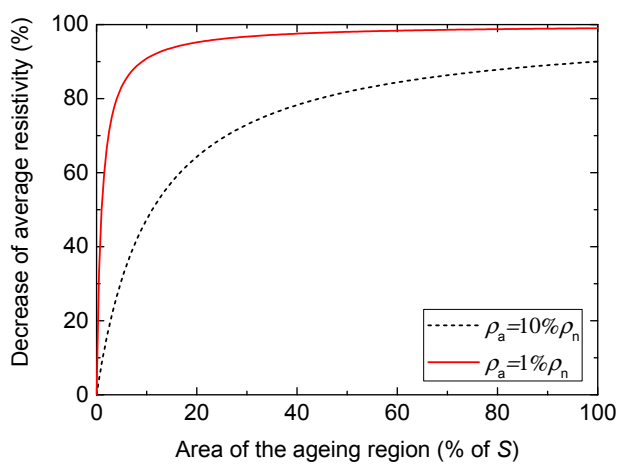

Figure 4. Decrease of the average resistivity with different percentages of the partial ageing region.

Actually, if the electric field applied on the ageing area is stronger than on other regions of paper, the change of average resistivity will be more obvious. Hence, it is even more sensitive for the ageing condition assessment of paper induced by electrical ageing.

\section{Iterative Inversion of Paper Resistivity}

Like DP measurements, it is destructive to take paper samples from transformers and measure their resistivities. Besides, the taken sample may not from the ageing region, according to the spatial distribution characteristic of paper ageing. Hence it is necessary to obtain the comprehensive resistivity through a non-destructive way.

With known resistivity values of oil and paper, the insulation resistances of transformer under different voltage applying method can be obtained through finite element simulation. When the resistivity of oil has a constant value, there is a fixed functional relationship between the resistivity of paper and insulation resistance:

$$
R=f(\rho)
$$

where $R$ is the insulation resistance, $\rho$ is the paper resistivity, and $f$ is the functional relationship between them.

As mentioned in Section 2.2, a change of paper resistivity will induce obvious changes in the measured voltage or current under a DC electric field. Conversely, the electrical quantities of transformer measured under a DC voltage can provide a good reflection of the paper resistivity. To illustrate this regulation, an axisymmetric model of transformer inner insulation structure was built as shown in Figure 5. The paper covering the windings and electrostatic rings were also considered in this model. By adopting different paper resistivity values, the corresponding insulation resistance results of this model obtained by finite element simulation were determined. During the simulation, the resistivity of oil was set as $10^{12} \Omega \cdot \mathrm{m}$. Two typical voltage application methods are considered.

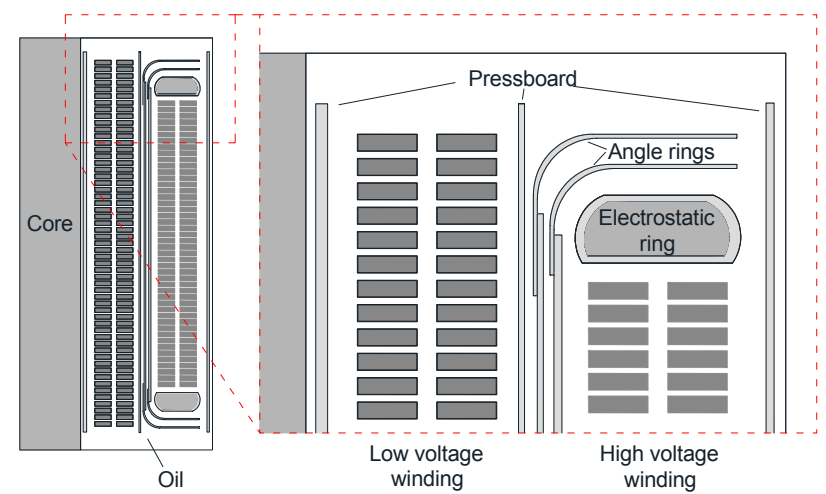

Figure 5. Axisymmetric model of transformer inner insulation structure. 
The equipotential lines obtained under the two typical voltage applying methods are shown as red lines in Figure 6. Most of the equipotential lines gathered inside the oil-impregnated paper. This distribution characteristic of equipotential lines proves the preceding analysis that the electric field in oil impregnated paper is much stronger than that in oil under DC voltage. Besides, the main parts of oil-impregnated paper surfaces were parallel to the equipotential lines, which verified the theory that certain area of oil impregnated paper can be regarded as some smaller areas of paper connected in parallel with each other, as stated in Section 2.2.

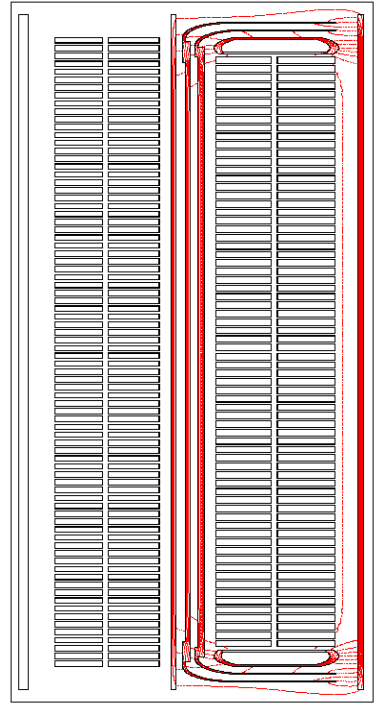

(a)

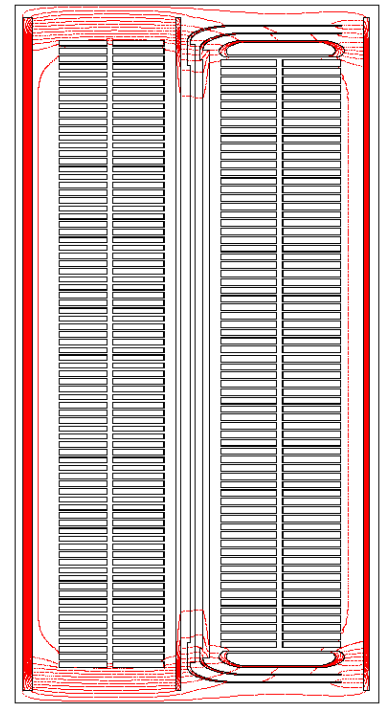

(b)

Figure 6. The equipotential lines under two typical DC voltage applying methods. (a) Voltage applied on high voltage winding; (b) Voltage applied on both windings.

Figure 7 shows the calculated insulation resistance results with different paper resistivity values. It is demonstrated that the relationship between $R$ and $\rho$ is nonlinear. As the function $f$ is monotonically continuous, as shown in Figure 7, an approximate value of $\rho$ can be obtained through iterative calculation. Although the analytic expression of $f$ is hard to obtain because of the complex insulation structure, this calculation process can be conducted through numerical simulation. Figure 8 shows the basic idea of this iterative calculation.

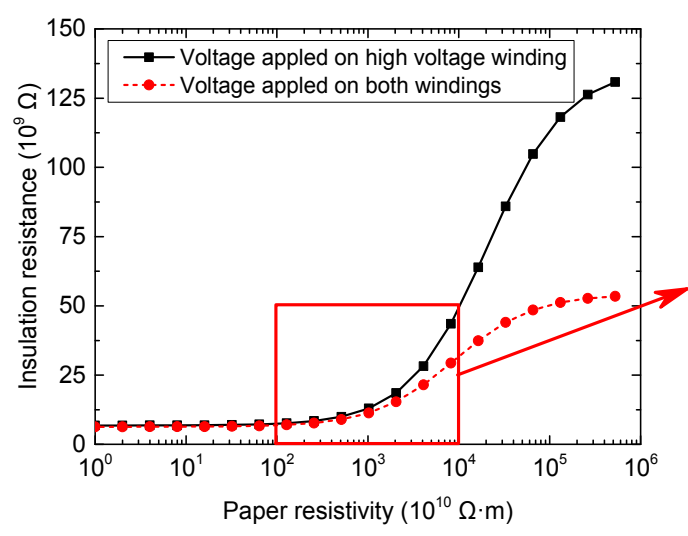

(a)

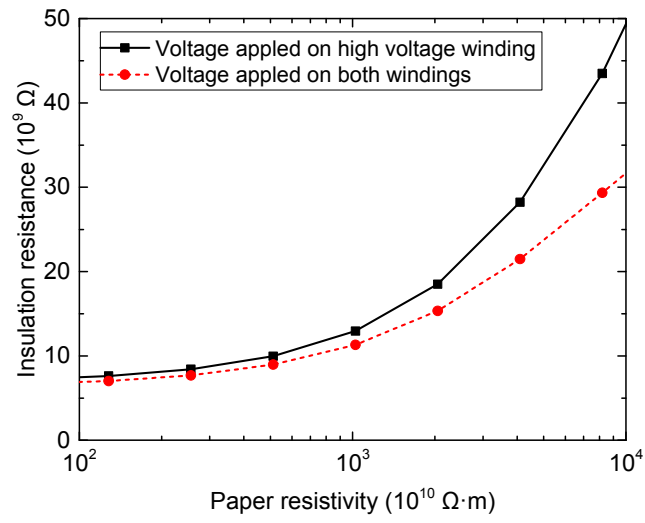

(b)

Figure 7. Insulation resistance results obtained using different paper resistivity values. (a) Paper resistivity varying between $10^{10} \sim 10^{16} \Omega \cdot \mathrm{m}$; (b) Local enlarged figure with paper resistivity varying between $10^{12} \sim 10^{14} \Omega \cdot \mathrm{m}$. 


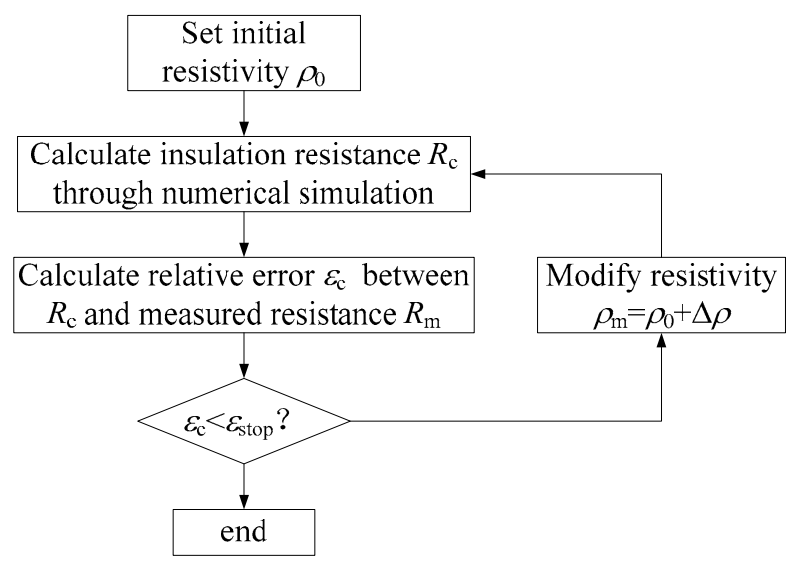

Figure 8. Basic idea of this iterative calculation.

Figure 6 also demonstrates that the function between $R$ and $\rho$ is also differentiable. For the iterative calculation of this kind of function, there are two typical methods, namely the bisection method and Newton's method.

\subsection{Bisection Method}

The bisection method is a solution-finding method that repeatedly bisects an interval and then selects the subinterval in which the solution must lies. The basic idea of this method is to minimize the interval which contains the solution.

For a transformer with known inner insulation structure, as well as the measured insulation resistance $R_{\mathrm{m}}$ and oil resistivity, the iteration of its paper resistivity follows the steps below:

(1) Set the initial interval $\left[\rho_{\min }, \rho_{\max }\right]$;

To ensure the actual paper resistivity $\rho$ belongs to this interval, Insulation resistance $R_{\max }$ and $R_{\min }$ are calculated using $\rho_{\max }$ and $\rho_{\min }$ separately. According to the monotone increasing relationship between paper resistivity and insulation resistance, $\rho \in\left[\rho_{\min }, \rho_{\max }\right]$ is equivalent to $R_{\mathrm{m}} \in\left[R_{\min }, R_{\max }\right]$. Hence, if $R_{\mathrm{m}} \in\left[R_{\min }, R_{\max }\right]$, then the initial interval $\left[\rho_{\min }, \rho_{\max }\right]$ is proper. Otherwise, enlarge the interval $\left[\rho_{\min }, \rho_{\max }\right]$ until $\left[R_{\min }, R_{\max }\right]$ meets the requirement.

(2) Calculate the middle point $\rho_{\mathrm{a}}$ of $\left[\rho_{\min }, \rho_{\max }\right] \cdot \rho_{\mathrm{a}}=\left(\rho_{\max }+\rho_{\min }\right) / 2$.

Firstly, calculate corresponding insulation $R_{\mathrm{a}}$, using $\rho_{\mathrm{a}}$. Then, calculate the relative error $\varepsilon_{\mathrm{c}}$ between $R_{\mathrm{a}}$ and $R_{\mathrm{m}}$. If $\varepsilon_{\mathrm{c}}$ is smaller than the preset termination error $\varepsilon_{\mathrm{stop}}$, then return $\rho_{\mathrm{a}}$ and stop iterating. Otherwise, turn to next step.

(3) Compare $R_{\mathrm{a}}$ and $R_{\mathrm{m}}$.

According to the compare result between $R_{\mathrm{a}}$ and $R_{\mathrm{m}}$, if $R_{\mathrm{a}}$ is larger than $R_{\mathrm{m}}$, set $\rho_{\max }$ equal to $\rho_{\mathrm{a}}$ and turn to Step 2, else, set $\rho_{\min }$ equal to $\rho_{\mathrm{a}}$ and turn to Step 2. A sufficiently precise value of $\rho$ will be obtained by performing the above steps until $\varepsilon_{\mathrm{c}}$ is smaller than $\varepsilon_{\text {stop }}$.

\subsection{Newton's Method}

When $f$ is differentiable, it can be expanded by Taylor series at $\rho_{0}$ as:

$$
R=f\left(\rho_{0}\right)+f^{\prime}\left(\rho_{0}\right) \cdot\left(\rho-\rho_{0}\right)+o\left[\left(\rho-\rho_{0}\right)^{2}\right]
$$


When ignoring the infinitesimal of higher order $o\left[\left(\rho-\rho_{0}\right)^{2}\right]$, the correction value $\Delta \rho$ of $\rho_{0}$ can be calculated according to the following equation:

$$
\Delta \rho=\rho-\rho_{0}=\frac{R-f\left(\rho_{0}\right)}{f^{\prime}\left(\rho_{0}\right)}
$$

Hence, with known insulation resistance $R=R_{\mathrm{m}}$, the corrected paper resistivity $\rho_{\mathrm{c}}$ can be obtained by:

$$
\rho_{\mathrm{c}}=\rho_{0}+\Delta \rho=\rho_{0}+\frac{R_{\mathrm{m}}-R_{0}}{f^{\prime}\left(\rho_{0}\right)}
$$

Sufficiently precise value will be obtained by repeatedly calculating the corrected value according to (5). This is the main idea of Newton's method.

For a transformer, the analytic expression of $f$ is hard to obtain. Hence differential calculation process is approximately substitute by difference calculation process as follows:

$$
f^{\prime}\left(\rho_{0}\right) \approx \frac{f\left(\rho_{0}+\delta \rho\right)-f\left(\rho_{0}\right)}{\delta \rho}
$$

where $\delta \rho$ is a tiny increase of $\rho$. The flowchart of Newton's method is shown as Figure 9.

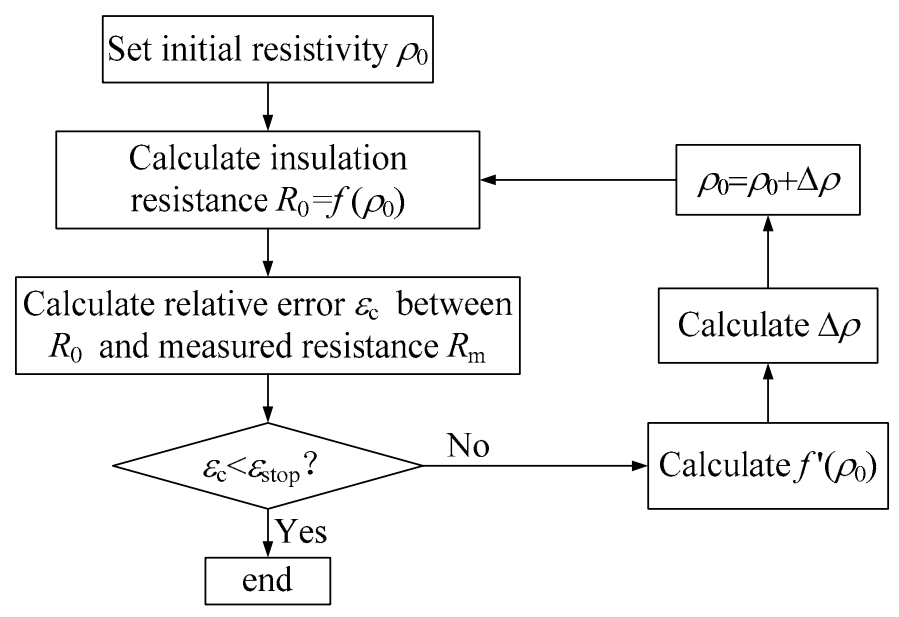

Figure 9. Flowchart of Newton's method for iterative calculation of paper resistivity.

\subsection{Comparison of Iterative Methods}

Figure 10 demonstrates the calculation results of each iterative step by using the bisection method and Newton's method separately. The axisymmetric model of a transformer in Figure 4 is taken as the object. The oil resistivity is $1 \times 10^{12} \Omega \cdot \mathrm{m}$, paper resistivity is $1 \times 10^{14} \Omega \cdot \mathrm{m}$. Both windings are connected and loaded with a high voltage while the core and the shell of the transformer are grounded, when obtaining the insulation resistance. The corresponding insulation resistance is $3.175 \times 10^{10} \Omega$. This value is taken as input during the iterative calculation. In the bisection method, the initial interval of paper resistivity is $\left[1 \times 10^{11} \Omega \cdot \mathrm{m}, 1 \times 10^{17} \Omega \cdot \mathrm{m}\right]$. In Newton's method, the values of initial resistivity $\rho_{0}$ are set as $10^{11} \Omega \cdot \mathrm{m}, 10^{13} \Omega \cdot \mathrm{m}, 10^{15} \Omega \cdot \mathrm{m}, 10^{17} \Omega \cdot \mathrm{m}$. The termination errors of both methods are all $0.1 \%$. 


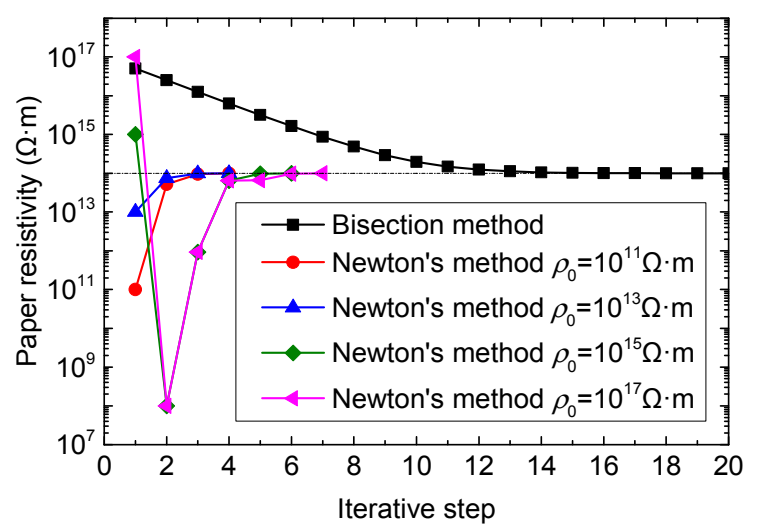

Figure 10. Calculation results using different iterative method.

For the iterative process, the bisection method converges more stably, while the results of Newton's method oscillate strongly. Besides, the iterative process of Newton's method is significantly influenced by the initial value of the iteration. Nevertheless, Newton's method has a much higher convergence speed than the bisection method. In Figure 10, it takes only 4 or 5 steps to reach a relative error lower than the termination error, while it takes as many as 20 steps for the bisection method.

According to the comparison, Newton's method is the first option for paper resistivity iteration for a transformer, in order to minimize the calculation time. Bisection can be regard as a complementary way to find a better initial value when Newton's method does not converge.

\section{Iterative Inversion of Paper Resistivity in Different Regions}

As mentioned before, the ageing of cellulose paper is not uniform. Hence, if the paper in transformer is divided into different regions, and corresponding paper resistivities are calculated separately, the accuracy will be improved to a considerable extent.

Usually more than one insulation resistance value can be obtained in practice. These insulation resistance values are obtained under different voltage application methods according to the structure of the transformer. These voltage application methods include: (i) High voltage windings are loaded with high voltage, while low voltage windings, core and shell of the transformer are grounded; (ii) low voltage windings are loaded with a high voltage, while the high voltage windings, core and shell of the transformer are grounded; (iii) both windings are connected and loaded with a high voltage while the core and shell of the transformer are grounded; etc.

With these different voltage application methods, the corresponding relationships between paper resistivity and insulation resistance can be built according to numerical simulation:

$$
\left\{\begin{array}{c}
R_{1}=f_{1}(\rho) \\
R_{2}=f_{2}(\rho) \\
\cdots \\
R_{n}=f_{n}(\rho)
\end{array}\right.
$$

In (10) $R_{1}, R_{2}, \ldots, R_{n}$ are the insulation resistance values obtained using different voltage application methods, and $f_{1}, f_{2}, \ldots, f_{n}$, are the corresponding relationships between the paper resistivity and insulation resistance.

In a transformer, paper resistivity and insulation resistance increase monotonically, as demonstrated before. Besides, the paper resistivity values in different regions have different contributions to the insulation resistance values, under different voltage application methods. Hence with $n$ insulation resistance values obtained from different voltage applying methods, $n$ paper 
resistivity values in different regions can be obtained from the following equations in theory. In each region, the resistivity of paper is assumed to be uniform:

$$
\left\{\begin{array}{c}
R_{1}=f_{1}\left(\rho_{1}, \rho_{2}, \cdots, \rho_{n}\right) \\
R_{2}=f_{2}\left(\rho_{1}, \rho_{2}, \cdots, \rho_{n}\right) \\
\cdots \\
R_{n}=f_{n}\left(\rho_{1}, \rho_{2}, \cdots, \rho_{n}\right)
\end{array}\right.
$$

For the iterative solution of nonlinear equations, the bisection method is too complicated and the advantage of Newton's method is more obvious. After expanding (11) by Taylor series at preset initial paper resistivity values $\left(\rho_{10}, \rho_{20}, \cdots, \rho_{n 0}\right)$, ignoring the infinitesimals of higher order, and transforming it into matrix form, the following equation can be obtained:

$$
J\left[\begin{array}{c}
\Delta \rho_{1} \\
\Delta \rho_{2} \\
\vdots \\
\Delta \rho_{n}
\end{array}\right]=\left[\begin{array}{c}
\Delta R_{1} \\
\Delta R_{2} \\
\vdots \\
\Delta R_{n}
\end{array}\right]
$$

where:

$$
\begin{aligned}
& \Delta \rho_{1}=\rho_{1}-\rho_{10} ; \Delta \rho_{2}=\rho_{2}-\rho_{20} ; \ldots ; \Delta \rho_{n}=\rho_{n}-\rho_{n 0} ; \\
& \Delta R_{1}=R_{1 \mathrm{~m}}-f_{1}\left(\rho_{10}, \rho_{20}, \ldots, \rho_{n 0}\right) ; \Delta R_{2}=R_{2 \mathrm{~m}}-f_{2}\left(\rho_{10}, \rho_{20}, \ldots, \rho_{n 0}\right) ; \ldots ; \Delta R_{n}=R_{n \mathrm{~m}}-f_{n}\left(\rho_{10}, \rho_{20}, \ldots, \rho_{n 0}\right) .
\end{aligned}
$$

$J$ is the Jacobian matrix expressed as:

$$
\boldsymbol{J}=\left[\begin{array}{cccc}
\frac{\partial f_{1}\left(\rho_{10}, \rho_{20}, \cdots, \rho_{n 0}\right)}{\partial \rho_{1}} & \frac{\partial f_{1}\left(\rho_{10}, \rho_{20}, \cdots, \rho_{n 0}\right)}{\partial \rho_{2}} & \cdots & \frac{\partial f_{1}\left(\rho_{10}, \rho_{20}, \cdots, \rho_{n 0}\right)}{\partial \rho_{n}} \\
\frac{\partial f_{2}\left(\rho_{10}, \rho_{20}, \cdots, \rho_{n 0}\right)}{\partial \rho_{1}} & \frac{\partial f_{2}\left(\rho_{10}, \rho_{20}, \cdots, \rho_{n 0}\right)}{\partial \rho_{2}} & \cdots & \frac{\partial f_{2}\left(\rho_{10}, \rho_{20}, \cdots, \rho_{n 0}\right)}{\partial \rho_{n}} \\
\vdots & \vdots & \ddots & \vdots \\
\frac{\partial f_{n}\left(\rho_{10}, \cdots, \rho_{n 0}\right)}{\partial \rho_{1}} & \frac{\partial f_{n}\left(\rho_{10}, \rho_{20}, \cdots, \rho_{n 0}\right)}{\partial \rho_{2}} & \cdots & \frac{\partial f_{n}\left(\rho_{10}, \rho_{20}, \cdots, \rho_{n 0}\right)}{\partial \rho_{n}}
\end{array}\right]
$$

Like (9), each element of $J$ is approximately obtained by a difference calculation process. For example:

$$
\frac{\partial f_{1}\left(\rho_{10}, \rho_{20}, \cdots, \rho_{n 0}\right)}{\partial \rho_{1}} \approx \frac{f_{1}\left(\rho_{10}+\delta \rho_{1}, \rho_{20}, \cdots, \rho_{n 0}\right)-f_{1}\left(\rho_{10}, \rho_{20}, \cdots, \rho_{n 0}\right)}{\delta \rho_{1}}
$$

where $\delta \rho_{1}$ is a tiny increase of $\rho_{1}$.

By solving (13), the correction values $\Delta \rho_{1}, \Delta \rho_{2}, \ldots, \Delta \rho_{n}$ for the preset paper resistivity values $\left(\rho_{10}, \rho_{20}, \ldots, \rho_{n 0}\right)$ can be obtained. Hence, the paper resistivity of each region is updated. Based on these updated paper resistivity values, corresponding insulation resistance values can be calculated, to compare with the measured values. Accurate paper resistivity values of each region can be reached by repeatedly updating the paper resistivity of each region until all the corresponding insulation resistance values are close enough to the given values.

\section{Application}

For simplicity, the axisymmetric model of a transformer illustrated in Figure 5 is still taken as the object in this section. Different paper resistivity values are applied to paper at different positions as shown in Figure 11 and Table 2. The pressboard at different locations is set with different resistivity values, in order to represent different insulation conditions. The papers with different resistivity values were filled with different colors, as shown in Figure 11. 


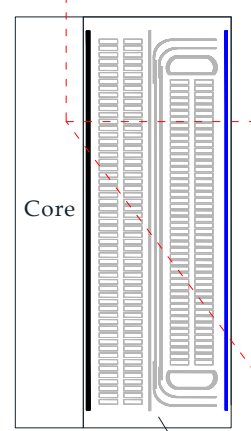

Oil

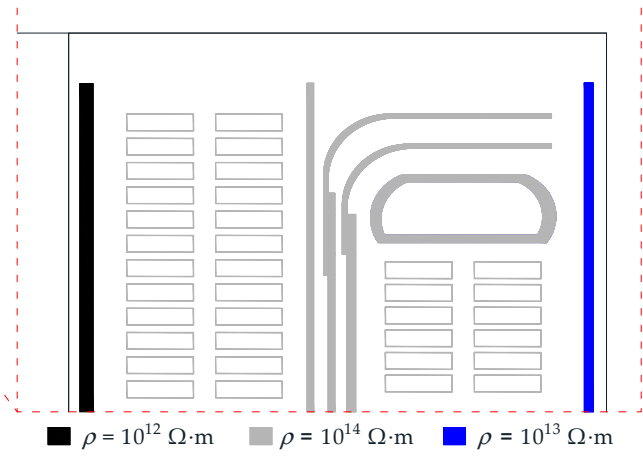

Figure 11. Setting of paper resistivity values and their corresponding location.

Table 2. Setting of paper resistivity values.

\begin{tabular}{cc}
\hline Location & Paper Resistivity $(\boldsymbol{\Omega} \cdot \mathbf{m})$ \\
\hline Pressboard between core and low voltage winding & $10^{12}$ \\
Pressboard between high voltage winding and shell & $10^{13}$ \\
Other paper and pressboard & $10^{14}$ \\
\hline
\end{tabular}

Three insulation resistance values under different voltage application methods were obtained through finite element analysis, with a fixed oil resistivity value of $10^{12} \Omega \cdot \mathrm{m}$. The obtained insulation resistance values are shown in Table 3.

Table 3. Insulation resistance values.

\begin{tabular}{ccc}
\hline \multicolumn{2}{c}{ Voltage Application Method } & Insulation Resistance $(\Omega)$ \\
\hline Loading with High Voltage & Grounding & \\
\hline High voltage windings & Low voltage windings, core and shell & $1.165 \times 10^{10}$ \\
Low voltage windings & High voltage windings, core and shell & $8.250 \times 10^{9}$ \\
High voltage windings and & Core and shell & $5.054 \times 10^{9}$ \\
low voltage windings & & \\
\hline
\end{tabular}

During the iterative inversion of paper resistivity, the oil resistivity and insulation resistance values in Table 3 are taken as the known quantities. The region division of paper is shown in Figure 12, where the oil impregnated papers in different regions were filled with different colors. It is obvious that papers in different regions have different contributions to the insulation resistances obtained under different voltage application methods. This is the main basis of the region division in this method.

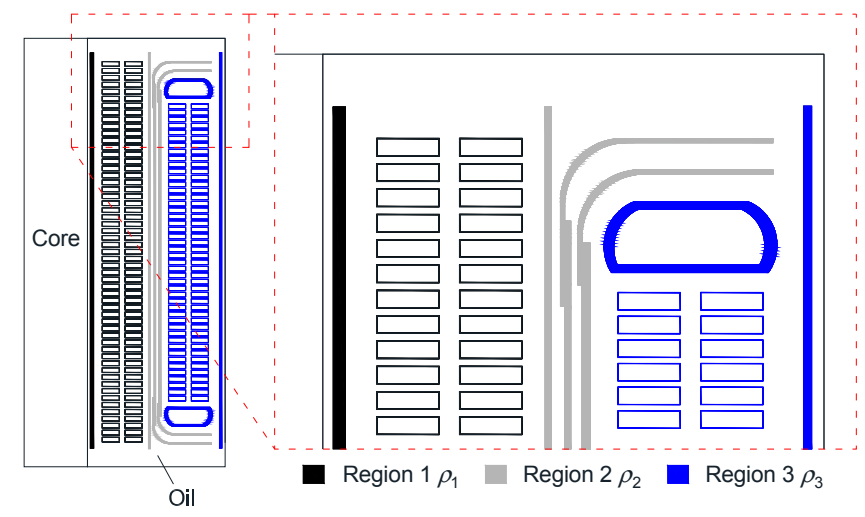

Figure 12. Region division of paper. 
It should be noted that there are differences between the region division in Figure 12 and the location of paper with different resistivities in Figure 11. In Figure 11, only the resistivities of some pressboards are changed. In Figure 12, all the papers in each region, including pressboard, paper covering the windings and electrostatic rings, and angle rings are considered to have the same resistivity. This difference is set to consider the actual situation, in which the specific locations of aged papers are unknown.

The iterative results of paper resistivity values for each region are shown in Figure 13. The termination error is also set as $0.1 \%$. In Figure 13, it takes 12 steps to reach the final results. The obtained paper resistivity values after iterative inversion are shown in Table 4 .

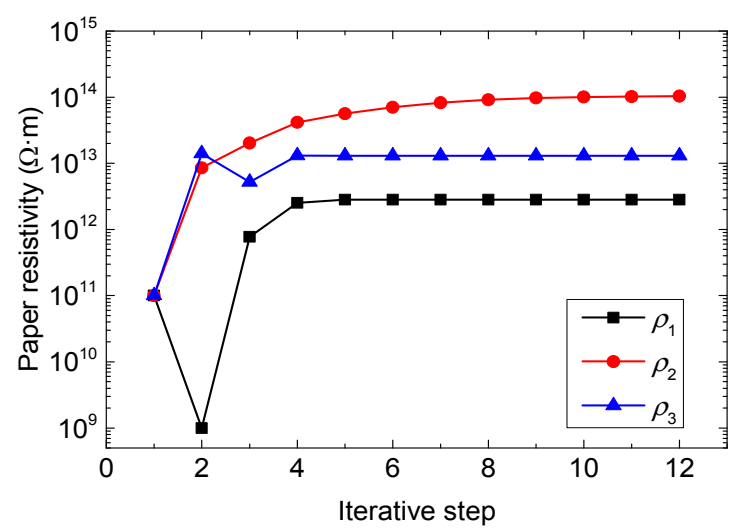

Figure 13. Iterative results of paper resistivity values for each region.

Table 4. Final results of paper resistivity iterative inversion.

\begin{tabular}{cc}
\hline Region & Paper Resistivity $(\boldsymbol{\Omega} \cdot \mathbf{m})$ \\
\hline Region 1 & $2.810 \times 10^{12}$ \\
Region 2 & $1.034 \times 10^{14}$ \\
Region 3 & $1.298 \times 10^{13}$ \\
\hline
\end{tabular}

The iterative results listed in Table 4 are slightly different from the preset values in Table 2 . Since the region division in Figure 12 is not exactly the same as the paper resistivity set in Figure 11, as a result, the obtained iterative results are "average" values. This "averaging" effect is the primary cause of the differences between the iterative results and the preset paper resistivity values.

The actual paper resistivity values (represented by the preset resistivities in Table 2) determine the insulation resistances of transformer (represented by the calculated insulation resistances in Table 3). Under fixed region division, the insulation resistances determine the calculated paper resistivities. Hence the results in Table 4 have the same variation tendency with the resistivity values in Table 2 . The more consistent the region division and actual location of paper with different resistivities are, the weaker the "averaging" effect is, and as a result, the closer the calculated resistivity values will be to the actual paper resistivity values.

In spite of these differences, the iterative results can still obviously demonstrate the change of paper resistivities in different region. Hence, the iterative method can not only help to obtain the aging condition of paper, but also demonstrate the aging distribution of paper to some extent.

In practice, the measured resistance values may not as precise as the data in Table 3 . To discuss the uncertainty in the resistance values, data in Table 4 were rounded off, and only one digit after the decimal point was kept, as shown in Table 5. Based on the same region division as shown in Figure 12, corresponding iterative results of paper resisitivities are obtained, as shown in Table 6 . The results in Table 6 do not have remarkable change compared with the data in Table 4, which demonstrates that the proposed iterative method is acceptable for practical application. 
Table 5. Rounded insulation resistance values.

\begin{tabular}{ccc}
\hline \multicolumn{2}{c}{ Voltage Application Method } & Insulation Resistance $(\Omega)$ \\
\hline Loading with High Voltage & Grounding & \\
\hline High voltage windings & Low voltage windings, core and shell & $1.2 \times 10^{10}$ \\
Low voltage windings & High voltage windings, core and shell & $8.3 \times 10^{9}$ \\
High voltage windings and & Core and shell & $5.1 \times 10^{9}$ \\
low voltage windings & & \\
\hline
\end{tabular}

Table 6. Iterative results with rounded insulation resistance values.

\begin{tabular}{cc}
\hline Region & Paper Resistivity $(\boldsymbol{\Omega} \cdot \mathbf{m})$ \\
\hline Region 1 & $2.760 \times 10^{12}$ \\
Region 2 & $1.042 \times 10^{14}$ \\
Region 3 & $1.359 \times 10^{13}$ \\
\hline
\end{tabular}

\section{Conclusions and Discussion}

The resistivity of oil impregnated paper will decrease during its aging process. According to this feature, this paper proposed a condition assessment method based on the changes of paper resistivity. Good power function relationships between DP and paper resistivity was found by analyzing the corresponding published experiment data, which proved the feasibility of this method. After analyzing the electric field distribution characteristic in transformer, the sensitivity of this method was discussed based on an idealized model.

This paper proposed a calculation method to obtain the resistivity value of paper in order to avoid direct sampling. This method was based on the idea of iterative inversion. Parameters such as oil resistivity and insulation resistance, which could be obtained without disassembling the transformer, were taken as the calculation input. Finite element simulation was used to build the relationship between insulation resistance and paper resistivity. Bisection method and Newton's method were compared, and Newton's method was selected as the first option of paper resistivity iteration for its faster convergence speed.

To promote the accuracy of paper resistivity calculation, this paper expanded the resistivity calculation method to multivariate iteration, based on Newton's method. The spatial distribution characteristic of paper aging were considered by dividing the oil impregnated paper in the transformer into different regions. The paper resistivities in different regions were obtained with insulation resistance values measured under different voltage application methods. After applying this multivariate iterative method to an axisymmetric model of a transformer, the sensitivity of the result was demonstrated, even if the region divisions were not exactly the same as the actual aging distribution.

Commonly, there are three kinds of insulation resistance values that can be obtained. This means that there can be as many as three regions and only three resistivity values can be obtained through multivariate iterative calculation. As a result, through three regions can consider the fact of non-uniform ageing and promote the accuracy of insulation condition assessment, the accuracy improvement is still somehow limited, hence, more input data are need to further improve this method. Generally speaking, resistivity has shown its potential in condition assessment of paper insulation. In the future, two work aspects will be done to improve this method. On the one hand, other non-destructive electrical measurement data, such as polarization loss under low frequency, will be used as an input of the multivariate iterative calculation, in order to further refine the region division and increase the calculation accuracy. On the other hand, a relationship between paper resistivity and insulation life will also be built to provide more guidance for the operation and maintenance of power transformers. 
Acknowledgments: This work was supported by the National Natural Science Foundation of China (NSFC, Grant No. 51477120).

Author Contributions: Jiangjun Ruan provided the main idea of this paper; Jin Shuo developed the method of paper resistivity iterative inversion; Jiangjun Ruan and Zhiye Du provided important guidance to this work; Yiming Xie, Lin Zhu and Yu Tian performed the calculation; Ruohan Gong, Guannan Li and Min Xiong analyzed the data; Shuo Jin wrote the paper.

Conflicts of Interest: The authors declare no conflict of interest.

\section{References}

1. Shroff, D.H.; Stannett, A.W. A Review of Paper Aging in Power Transformers. IEE Proc. C 1985, 132, 312-319. [CrossRef]

2. Saha, T.K. Review of Time-domain Polarization Measurements for Assessing Insulation Condition in Aged Transformers. IEEE Trans. Power Deliv. 2003, 18, 1293-1301. [CrossRef]

3. Lin, Y.; Yang, L.; Liao, R.; Sun, W.; Zhang, Y. Effect of Oil Replacement on Furfural Analysis and Aging Assessment of Power Transformers. IEEE Trans. Dielectr. Electr. Insul. 2015, 22, 2611-2619. [CrossRef]

4. Krause, C. Power Transformer Insulation-History, Technology and Design. IEEE Trans. Dielectr. Electr. Insul. 2012, 19, 1941-1947. [CrossRef]

5. Martin, D.; Cui, Y.; Ekanayake, C.; Ma, H.; Saha, T. An Updated Model to Determine the Life Remaining of Transformer Insulation. IEEE Trans. Power Deliv. 2015, 30, 395-402. [CrossRef]

6. Wang, M.; Vandermaar, A.J.; Srivastava, K.D. Review of Condition Assessment of Power Transformers in Service. IEEE Electr. Insul. Mag. 2002, 18, 12-25. [CrossRef]

7. Arshad, M.; Islam, S.M. Significance of Cellulose Power Transformer Condition Assessment. IEEE Trans. Dielectr. Electr. Insul. 2011, 18, 1591-1598. [CrossRef]

8. Ali, M.; Eley, C.; Emsley, A.M.; Heywood, R.; Xaio, X. Measuring and Understanding the Ageing of Kraft Insulating Paper in Power Transformers. IEEE Electr. Insul. Mag. 1996, 12, 28-34. [CrossRef]

9. Jin, S.; Ruan, J.; Du, Z.; Yang, Z.; Zhou, T.; Huang, G.; Zhu, L. Charge Transport in oil Impregnated Paper Insulation Under Temperature Gradient Using Transient Upstream FEM. CSEE J. Power Energy Syst. 2015, 1, 3-8.

10. Jin, S.; Ruan, J.; Du, Z.; Huang, G.; Zhu, L.; Guan, W.; Yang, Z.; Li, L. Charge Transport Simulation in Single-Layer Oil-Paper Insulation. IEEE Trans. Magn. 2016, 52, 8101404.

11. Sun, H.C.; Huang, Y.C.; Huang, C.M. A Review of Dissolved Gas Analysis in Power Transformers. Energy Procedia 2012, 14, 1220-1225. [CrossRef]

12. Pradhan, M.K.; Ramu, T.S. On the Estimation of Elapsed Life of Oil-immersed Power Transformers. IEEE Trans. Power Deliv. 2005, 20, 1962-1969. [CrossRef]

13. Mcnutt, W.J. Insulation Thermal Life Considerations for Transformer Loading Guides. IEEE Trans. Power Deliv. 1992, 7, 392-401. [CrossRef]

14. Frimpong, G.K.; Oommen, T.V.; Asano, R. A Survey of Aging Characteristics of Cellulose Insulation in Natural Ester and Mineral Oil. IEEE Electr. Insul. Mag. 2011, 27, 36-48. [CrossRef]

15. Emsley, A.M.; Stevens, G.C. Review of Chemical Indicators of Degradation of Cellulosic Electrical Paper Insulation in Oil-filled Transformers. IEE Proc. Sci. Meas. Technol. 1994, 141, 324-334. [CrossRef]

16. Emsley, A.M.; Xiao, X.; Heywood, R.J.; Ali, M. Degradation of Cellulosic Insulation in Power Transformers. Part 2: Formation of Furan Products in Insulating Oil. IEE Proc. Sci. Meas. Technol. 2000, 147, $110-114$. [CrossRef]

17. Kachler, A.J.; Hohlein, I. Aging of Cellulose at Transformer Service Temperatures. Part 1: Influence of Type of Oil and Air on the Degree of Polymerization of Pressboard, Dissolved Gases, and Furanic Compounds in Oil. IEEE Electr. Insul. Mag. 2005, 21, 15-21. [CrossRef]

18. Matharage, S.Y.; Liu, Q.; Wang, Z.D. Aging Assessment of Kraft Paper Insulation through Methanol in Oil Measurement. IEEE Trans. Dielectr. Electr. Insul. 2016, 23, 1589-1596. [CrossRef]

19. Mineral Oil-Impregnated Electrical Equipment in Services-Guide to the Interpretation of Dissolved and Free Gases Analysis; IEC-60599-2007; IEC: Geneva, Switzerland, 2007.

20. IEEE Guide for the Interpretation of Gases Generated in Oil-Immersed Transformers-Redline; IEEE Std C57.104-2008; IEEE: New York, NY, USA, 2009. 
21. Sharma, N.K.; Tiwari, P.K.; Sood, Y.R. Review of Artificial Intelligence Techniques Application to Dissolved Gas Analysis on Power Transformer. Int. J. Comput. Electr. Eng. 2011, 3, 577-582. [CrossRef]

22. Yang, M.T.; Hu, L.S. Intelligent Fault Types Diagnostic System for Dissolved Gas Analysis of Oil-immersed Power Transformer. IEEE Trans. Dielectr. Electr. Insul. 2013, 20, 2317-2324. [CrossRef]

23. Abu-Siada, A.; Lai, S.P.; Islam, S.M. A Novel Fuzzy-Logic Approach for Furan Estimation in Transformer Oil. IEEE Trans. Power Deliv. 2012, 27, 469-474. [CrossRef]

24. Hadjadj, Y.; Fofana, I.; van de Voort, F.R.; Bussieres, D. Potential of Determining Moisture Content in Mineral Insulating Oil by Fourier Transform Infrared Spectroscopy. IEEE Electr. Insul. Mag. 2016, 32, 34-39. [CrossRef]

25. Saha, T.K. Review of Modern Diagnostic Techniques for Assessing Insulation Condition in Aged Transformers. IEEE Trans. Dielectr. Electr. Insul. 2003, 10, 903-917. [CrossRef]

26. Cho, J.S.N.; Fofana, I.; Hadjadj, Y.; Beroual, A. Review of Physicochemical-Based Diagnostic Techniques for Assessing Insulation Condition in Aged Transformers. Energies 2016, 9, 367.

27. Linhjell, D.; Lundgaard, L.; Gafvert, U. Dielectric Response of Mineral Oil Impregnated Cellulose and the Impact of Aging. IEEE Trans. Dielectr. Electr. Insul. 2007, 14, 156-169. [CrossRef]

28. Gao, J.; Yang, L.; Wang, Y.; Qi, C. Quantitative Evaluation of Ageing Condition of Oil-paper Insulation Using Frequency Domain Characteristic Extracted from Modified Cole-cole Model. IEEE Trans. Dielectr. Electr. Insul. 2015, 22, 2694-2702. [CrossRef]

29. Yousof, M.F.M.; Ekanayake, C.; Saha, T.K. Examining the Ageing of Transformer Insulation Using FRA and FDS Techniques. IEEE Trans. Dielectr. Electr. Insul. 2015, 22, 1258-1265. [CrossRef]

30. Wolny, S. Aging Degree Evaluation for Paper-oil Insulation Using the Recovery Voltage Method. IEEE Trans. Dielectr. Electr. Insul. 2015, 22, 2455-2462. [CrossRef]

31. Sarkar, S.; Sharma, T.; Baral, A.; Chatterjee, B.; Dey, D.; Chakravorti, S. An Expert System Approach for Transformer Insulation Diagnosis combining Conventional Diagnostic Tests and PDC, RVM Data. IEEE Trans. Dielectr. Electr. Insul. 2014, 21, 882-891. [CrossRef]

32. Gubanski, S.M.; Boss, P.; Csepes, G.; Der, H.V.; Guuinic, J.F.P.; Gäfvert, U.; Karius, V.; Lapworth, J.; Urbani, G.; Werelius, P. Dielectric response methods for diagnostics of power transformers. IEEE Electr. Insul. Mag. 2003, 19, 12-18.

33. Liao, R.; Hao, J.; Chen, G.; Yang, L. Quantitative Analysis of Ageing Condition of Oil-paper Insulation by Frequency Domain Spectroscopy. IEEE Trans. Dielectr. Electr. Insul. 2012, 19, 821-830. [CrossRef]

34. Wang, S.; Zhang, G.; Wei, J.; Yang, S.; Dong, M.; Huang, X. Investigation on Dielectric Response Characteristics of Thermally Aged Insulating Pressboard in Vacuum and Oil-impregnated Ambient. IEEE Trans. Dielectr. Electr. Insul. 2010, 17, 1853-1862. [CrossRef]

35. Saha, T.K.; Purkait, P. Investigation of an expert system for the condition assessment of transformer insulation based on dielectric response measurements. IEEE Trans. Power Deliv. 2004, 19, 1127-1134. [CrossRef]

36. Setayeshmehr, A.; Fofana, I.; Eichler, C.; Akbari, A. Dielectric Spectroscopic Measurements on Transformer Oil-paper Insulation Under Controlled Laboratory Conditions. IEEE Trans. Dielectr. Electr. Insul. 2008, 15, 1100-1111. [CrossRef]

37. Han, H. Research on Transformer Aging Character and Analysis Aging Mechanism. Master's Thesis, Changsha University of Science and Technology, Changsha, China, 2007.

38. Prevost, T.A.; Oommen, T.V. Cellulose Insulation in Oil-filled Power Transformers: Part I-History and Development. IEEE Electr. Insul. Mag. 2006, 22, 28-35. [CrossRef]

(C) 2017 by the authors. Licensee MDPI, Basel, Switzerland. This article is an open access article distributed under the terms and conditions of the Creative Commons Attribution (CC BY) license (http://creativecommons.org/licenses/by/4.0/). 